\title{
A remark on compact semigroups having certain decomposition spaces embeddable in the plane
}

\author{
R. P. Hunter and L. W. And erson
}

\begin{abstract}
Let $S$ be a compact connected semigroup. If the decomposition space of $S$, under the action of a compact connected subgroup at the identity, is a plane continuum then this decomposition is a congruence.
\end{abstract}

Let $S$ be a compact semigroup with identity 1 and $H_{1}$ the maximal subgroup at 1 . In general, the orbits $x H_{1}$ do not form a congruence on $S$ as various examples of semi-direct products show.

In [2] it is shown that if $G$ is a compact connected subgroup of $H_{1}$ such that $S / G$ is one dimensional then the orbits of $G$ form a congruence. Such an $S$ must then contain a local thread lying in the centralizer of $G$.

We remark here that the same result holds if $S / G$ is a plane continuum. It should be noted that there exist one dimensional compact connected semigroups with identity and zero which are not plane continua [4].

PROPOSITION. Let $S$ be a compact connected semigroup with identity and let $G$ be a compact connected subgroup of $H_{1}$ such that $S / G$, the hyperspace of orbits $x G$ is embeddable in the plane. Then these orbits form a congmence. Moreover $S$ contains a local thread at 1 which lies in the centralizer of $G$. 
Indication of proof. If we do not have $G x \subseteq x G$ for all $x \in S$, which is the condition that the orbits form a congruence, then $G$ can be considered as a transformation group of the plane continuum $S / G$, the action being, of course, $g\{x G\}=\{g x G\}$. If the orbit $G \bar{x}$ (where $\bar{x}=\{x G\}$ ) is non-degenerate it must be a simple closed curve. Now by arguments quite similar to those employed in [4] or [1] it follows that given two non-degenerate orbits $G \bar{x}_{0}$ and $\overline{G x}$, then one of these simple closed curves must enclose the other. Next we note that a non-degenerate orbit say, $\bar{G} \bar{t}$ unioned with that portion of $S / G$ lying in the unbounded complementary domain of $\bar{G} \bar{t}$, is a subcontinuum $S_{\bar{t}}$ of $S / G$ filled up in a continuous manner by the orbits. In particular, the union of the non-degenerate orbits of points of $S_{\bar{t}}$ is a closed subset of $S_{\bar{t}}$. The degenerate orbits clearly form a closed set. Since $S_{\bar{t}}$ is a continuum all this is impossible were there to exist a degenerate orbit defined by a point of $S_{\bar{t}}$. Thus a degenerate orbit must be enclosed by every non-degenerate one, and, in particular $G \bar{l}=\{\bar{l}\}$ is enclosed by every non-degenerate orbit. Since the collection of orbits is a continuous collection it follows that every orbit save $G \bar{I}$ is non-degenerate. (Every non-degenerate orbit must enclose yet another.) In fact, under the supposition that the orbits did not form a congruence, we see that $S / G$ is now a disc with $\bar{l}$ as an interior point.

It now follows that $K / G$ separates the plane. However, $S / K$, the the maximal ideal of $S$. However, $S / K$, the Rees quotient, acts on $S / G / T$, where $T$ is the decomposition formed by collapsing $K / G$ to a point. All of this is impossible since the latter space is a sphere or a sphere tangent to a disc and cannot be acted upon by a compact connected semigroup with identity and zero.

Thus the natural map $\rho: S+S / G$ is a continuous (open) homomorphism of $S$ onto a plane semigroup. By the proposition of [5] we know that $S / G$ contains a local thread at the identity, say $I$. Considering the closure of $\rho^{-1}(I)$ the desired local thread exists, arguing as in [2], [3]. 


\section{References}

[1] L.W. Anderson and R.P. Hunter, "The H-equivalence in compact semigroups", Bulz. Soc. Math. Belg. 14 (1962), 274-296.

[2] L.W. Anderson and R.P. Hunter, "Compact semigroups having certain one dimensional hyperspaces", Amer. J. Math. (to appear).

[3] Karl Heinrich Hofmann and Paul S. Mostert, Elements of compact semigroups (Charles E. Merrill Books, Colombus, Ohio, 1966).

[4] R.P. Hunter, "Certain upper semi-continuous decompositions of a semigroup", Duke Math. J. 27 (1960), 283-290.

[5] R.P. Hunter and L.W. Anderson, "Certain homomorphisms of a compact semigroup onto a thread", J. Austral. Math. Soc. 7 (1967), $311-322$.

Pennsylvania State University,

University Park,

Pennsylvania, USA. 